\title{
CONTEXT AWARE COLLABORATION IN ENTERPRISES
}

Keywords: Context, Collaboration, Agents

\begin{abstract}
Providing the most relevant information at the most appropriate time at the most appropriate location helps in improving the overall enterprise productivity. Contextual information plays a role in achieving this objective. Richer and deeper the context is, higher is the relevance and appropriateness. In this paper, we discuss the various aspects of a context and the ways and means of tracking the same so as to exploit the most recent and expectedly accurate description of the business situation in delivering the information to assist in collaboration. Further, we discuss the role of data and app grids in meeting the real-time delivery requirements.
\end{abstract}

\section{INTRODUCTION}

Advancements in technologies enable communication, coordination, and cooperation mechanisms. Within an organization, the major challenges are how to shorten the product development life cycle; and how to manage the entire cycle from conception to product development, sales and maintenance at lower costs by effective usage of technology. Also, competition poses severe time constraints - a new product, without any compromise on quality should be launched before a competitor does. It is in this effort, to build products and services of highest quality in challenging time period and lower production costs that enterprises need to collaborate with partners / suppliers (O'Keefe et al 2001, Blois K et al 1998). With advancement of technology as the driving force, virtual collaborations are increasingly being adapted across enterprises (WorldCom, 2001). Several commercial collaboration tools are available (eRoom, NetMeeting).

While the utilities of intra-organization and interorganization collaboration activities are desirable, there are several factors that impede its practice. These factors comprise both technological as well as social issues. Heterogeneity - in communication networks, communication devices, and information sources across organizations imposes challenges on creating effective collaboration technology. Also, collaboration essentially requires large-scale video and audio conferencing, transcoding to facilitate communication through devices with diverse characteristics, and such applications need high computational resources. Further, with advancement in mobile communication, service representatives who are frequently travelling require coordinated collaboration across departmental and corporate boundaries for sustaining competitive advantage and reducing business processing cycle times. As a result, large enterprises are challenged to simultaneously provide a unified solution to support enterprise integration, mobile applications, and collaboration. Such technological complexities in large-scale multi-party collaboration could be handled using grid technologies (Hinde $\mathrm{S}$ et al 2002 ). Grid technology (Karl et al 2001 ) provides highly efficient resource sharing mechanisms in addition to security, reliability, and fault tolerant mechanisms in heterogeneous resource environments. Data grid (Chervenak A et al 1999, Avaki 2003) provides an efficient, scalable access to real-time data that is globally distributed and in different formats. Data and computational grids provide an easy way of handling heterogeneity issues as well as computational issues. 
Technology enabled on-line collaboration requires systems that keep track of all activities, often realtime events - providing real-time visibility and control of information by the collaborators involved. Technology provides several support systems like ERP, CRM, and several workflow systems to coordinate tasks that aid enterprises in procedurally oriented transactions. These systems coordinate tasks, but do not provide support for individuals or groups working with them.

Effective knowledge sharing enhances the efficiency of collaboration. Perkins et al (Perkins et al 2003), outline different characteristics of collaboration activities that are enhanced by effective knowledge management as degree of trust, correlation with common goals, personal relationships, expectation of collaborating parties, and required competence. A system that provides knowledge management through provisioning of information to facilitate effective collaboration through information sharing amongst the collaborators must be "aware" of users" as well as the business context. Context could be defined as any information that is used to describe the user's environment (Abowd, G.C. et al, 1999). Context specific information, both in content as well as form, enhances the team's efficiency by creating awareness of the collaboration activity amongst all of the collaborators. Kammanahalli et al (Kammanahalli $\mathrm{H}$ et al 2003) have discussed a system, that facilitates effective interactions during a synchronous collaborative session by using proactive triggering based on current context information.

This paper highlights our solutions to address effective context relevant knowledge management through a system sitting on top of the grid architecture, comprising data and app grids, to facilitate effective collaboration. Since information is accessed from different business scenarios there is a need for contextual information. The knowledge sharing among collaborators is achieved through multiple collaborating agents that are specifically related to the various aspects of a context in an enterprise.

\section{CONTEXT IN ENTERPRISES}

A typical user and data perspective of an enterprise is depicted in Figs. 1 and 2 (All figures are provided towards the end of the paper). An overall network of an enterprise (a) covering globally distributed offices, and (b) to provide global access to enterprise resources for its employees comprises multiple, heterogeneous sub-networks. Fig. 2 depicts the distribution of data over these sub-networks and in order to provide efficient, real-time, and ubiquitous access to data, it is appropriate to set up dedicated data grids. The figure indicates two distinct kinds of grids, namely, Department data grids and Enterprise data grid. Department data grids are specific to the different locations of the enterprise and the enterprise data grid interconnects these multiple Department data grids to form a single, connected data network. Data grids are useful especially during audio and videoconferences demanding real-time access to the globally distributed data. We propose multiple, collaborating agents to provide contextaware access to the data stored in data grids. The agents interact with the data grid managers to efficiently retrieve data. In the following, we provide a brief description of an enterprise context to help provide the most relevant information at the most appropriate time to the staff of the enterprise. Context, as described below, consists of two parts, namely, business context and user context.

Business Context (BC): During collaboration across an enterprise, it is very important to integrate information sources that capture all the activities of the domain across the organization. This integration provides different views of the enterprise, relating relevant tasks to be performed by the tools that support them and also, establishing connections between the information sources themselves. Enterprise ontology provides a set of terms covering the various activities of the enterprise. Ontology, that is a communication medium, helps in resolving the ambiguities and creating shared understanding of relevant aspects of business enterprise. Ontology, in an enterprise, contains definitions of several business aspects that include information about people and also information about computational systems across the enterprise. The complete list of enterprise ontology as given by AIAI (The Enterprise Ontology, Uschold $M$ et al 1998) can form the basis for the definition of Business Context so as to succinctly capture the current business situation.

User Context (UC): User's context represents the user's current situation. UC contains user's Current context (CC), defined by the user's current activity and the entities associated with it, and analyzed context (AC) gives detailed information about user preferences. AC is generated by analyzing and aggregating user's past contexts.

We consider an activity as a discrete event, for example, receiving an email or attending a meeting. Each context is associated with the following entities: activity being performed, user identity, 
user's role, location of the user, data being accessed, device being used, time, network communication channel, and network condition.

Together with business context and user's context, the entire enterprise context is captured. Business context provides the most recent and relevant information, from all of the concerned information sources in the current business situation amongst the collaborators, while user's context provides information about which business information is relevant to the user and how best it could be presented to the user.

An example of an enterprise context is depicted in Fig. 3. Note that "Vendor Rating" indicates an activity that is a part of purchase orders processing undertaken by a staff playing the role of purchase manager. Also, an illustration of user context is that an R\&D manager sending an email related to a pending purchase requisition. Fig. 4 describes some explicit means of capturing context information. There are six different categories: Sensory data based, Presence data based, Workflow data based, Activity data based, Agenda data based, and Goal data based categories. For each of these categories, we have provided several possible ways of obtaining the context information.

\section{CONTEXT IDENTIFICATION}

In order to provide the most relevant information at the most appropriate time for a staff of an enterprise, it is essential to track the context associated with the staff on a continuous basis. This is achieved by separately tracking each of the context categories. A generic, feedback-oriented context identification approach is depicted in Fig. 5.

Observe that the contexts related to the business aspects and user-specific aspects are separately extracted and combined to arrive at an enterprise context. Specifically, when a staff participates in a meeting, the current context, identified and associated with the staff, will be used to extract the information that will be useful during collaboration activity such as meeting. Furthermore, the continuous tracking means that even during the meeting, as meeting progresses, context is changed to reflect the current business situation, and hence, any additional information that becomes relevant on account of the changed context is obtained and provided on the user terminal.

Business context is obtained based on (a) nature of query inputted by the staff; (b) active tasks part of a workflow; and (c) business schema providing relationship among various business objects and mapping between tasks of a workflow and the business objects.

User context is obtained based on (a) the current role of a staff; note that a staff can play multiple roles at different times; (b) the current designation; this plays a role in the kind of information that can be accessed and provided; (c) the user profile indicating the user-specific requirements; and (d) the user activities that help relevance-based ordering of the information.

Our approach to context-based retrieval is based on aggregating a past sequence of individual contexts and associating the current user activity to those past contexts to determine the best way to deliver information relevant to the current activity. Due to the continuity of user activities aimed at achieving a certain goal, continuity in contexts could be established and exploited for determining and retrieving more relevant information. This aggregation is achieved making use of the repetitive patterns inherent in the activities performed; for example, consider a sequence of email exchanges amongst the members of a team. Analysis of this sequence of emails is likely to reveal the common thread across those emails. Likewise, analyzing consecutive meeting minutes could provide contextual information about the current meeting. Context information derived by recognizing document access patterns has also been explored by Bauer (Bauer et al 2001). Bauer et al propose WordSieve, a system where the context of a user's current interest is derived by analyzing the user's web page access patterns. Past context sequences are analyzed and aggregated to derive usage trends and preferences, which is used to obtain a user's AC (Kammanahalli et al 2004).

In summary, by analysing and aggregating past individual contexts, the implicit information and delivery needs of users pursuing a particular activity and the format of presentation of this information in the current context can be captured.

\section{MULTI-AGENT COLLABO- RATION}

We identify distinct agents for each of the context categories described in the previous section. There are basically six broad categories resulting six class of agents: $\mathrm{S}$ agents for tracking sensors, $\mathrm{P}$ agents for tracking user presence on a network, $\mathrm{W}$ agents for 
tracking user activities with respect to a workflow, A agents for tracking user activities, $\mathrm{N}$ agents for tracking agenda items, and $\mathrm{G}$ agents for tracking a goal. Agelets (Slets, Plets, and so on), instantiated version of these agents, are distributed over the enterprise network and they constantly track and provide the changed information to their respective servers. This is depicted in Fig. 6. Observe the both raw data and the analysis results are store in a distributed manner over the enterprise network. For example, SDB corresponds to sensory data and PDB corresponds to presence data (refer to Fig. 6). Agelets can be very thin and can just provide the raw data to the servers. However, in certain cases, especially to reduce the load on the enterprise network, the agelets can undertake some preprocessing before communicating the data to the servers. We further discuss this issue later in this section.

The agents described above exhibit some characteristics with respect to persistence and mobility. A brief characterization of some of the agents is provided in Fig. 7. Some illustrative procedures for context tracking are described in Fig. 8.

In order for agelets to undertake some preprocessing, the data and the available applications within the enterprise are distributed over data grid and app grid respectively. Observe that the app grid contains the enterprise application resources for sharing efficiently the same across the enterprise. Such paired grid architecture helps agelets (that need to operate in real-time) to obtain raw data, get additional data from the data grid, use the appropriate application for pre-processing and required level of analysis, and finally communicate the results to the appropriate DS for context tracking purposes. This interaction at a high level is depicted in Fig. 9.

The collaboration among multiple agents can be either explicit (that is, collaboration in response to a user request) or implicit (that is, collaboration to achieve a system task). An example of these two kinds of collaborations is provided in Fig. 10.

\section{DISCUSSION AND CONCLUSION}

There are several business scenarios wherein people involved collaborate and negotiate to arrive at a decision. In such situations, providing the right information for each of the participants helps carry out their tasks more effectively thereby enhancing the overall enterprise productivity. Considering a business scenario in which a group consisting of a purchase manager, an R \& D manager, and a finance manager are meeting to negotiate on the procurement of items necessary for a research project requires that the purchase manager is well prepared to answer the queries from both the R \& D manager and the finance manager. The required information to help the purchase manager is obtained both from the internal information sources and the external information sources. As described in this paper, both business context (related to purchase of some items, related vendor information, information such as indigenous or to be imported, and other information as appropriate from the workflow) and user context (related to the role and designation, study carried out by reading the related documents and product brochures, relevant emails exchanged on the subject matter, meeting location, and the preferred device for providing real-time information) are used to retrieve the most relevant information from the multiple information sources. Further, as the meeting progresses and issues get discussed in depth, the context changes and additional information needs to be retrieved and provided to enable the purchase manage to continue to be effective. To achieve this real-time provisioning of information, that may also involve real-time analysis to be performed, combined data and app grids seem to be very appropriate.

In this paper, we have discussed the various aspects of the notion of context in an enterprise and have described approaches for tracking the various aspects of the context in order to provide the most relevant information at the most appropriate time.

We are working on extending a presently under development system for enterprise related business scenarios. The objective is to link the system with the enterprise information system and track context at individual, department, and enterprise levels as business situations change. Further, we are planning on extending the system to assist the participants of an internal meeting in collaboration and negotiation so as to reduce the overall negotiation effort in arriving at a mutually agreeable decision. An initial version of such a system is depicted in Fig. 11. Note that such a system makes a video conferencing as effective as a face-to-face meeting. 


\section{REFERENCES}

Abowd, G.C., A.K. Dey, P.J. Brown, N. Davies. M. Smith and P. Steggles., 1999. "Towards a better understanding of context and context-awareness," Handheld and Ubiquitous Computing, (H.W. Gellersen, Ed.), Springer, Berlin, pp. 304-307

Avaki., 2003. Keep it Simple - Overcome Information integration challenges with Avaki Data Grid Software, http:// www.avaki.com/papers

Bauer, T and Leake D., 2001. "Exploiting information access pattern for context-based retrieval," Proceedings of the 2002 International Conference on Intelligent User Interfaces, ACM Press.

Blois K, 1998. "A trust interpretation of business to business relationships: a case study discussions", Management Decisions, pp $302-308$

Chervenak A, Foster I, Kesselman C, Salisbury C, and Turke S., 1999. "The Data Grid: Towards an Architecture for the Distributed Management and Analysis of Large Scientific Datasets", http://www.globus.org.

eRoom, http://www.documentum.com

Hinde S and Wilcock L., 2002. "Gird as a platform for communication, collaboration and e-science", Technical Report, HP Labs, http://www.hpl.hp.com/techreports/2002/HPL-2002125.pdf

Kammanahalli H, Gopalan S, Sridhar V, and Ramamrithm K., 2003. "CART: An Information System for ContextAware Information Access during Synchronous Webbased Collaborations", $2^{\text {nd }}$ International Conference on Communication, Internet and Information Technology, November 2003, Scotsdale, USA

Kammanahalli H, Gopalan S, Sridhar V, and Ramamrithm
K., 2004. "Context Aware Retrieval in Web-based Collaboration, submitted to Context Modelling and Reasoning Workshop, March 2004, Florida, USA

NetMeeting, http://www.microsoft.com

O'Keefe ., 2001. "Building intellectual capital in the supply chain - the role of ecommerce", Supply Chain Management, pp 148 - 151

Perkins J, Jorgensen A, Burton L and Cox S., 2003. "Building Supply Chain Relationships with Knowledge Management", Proceeding of $5^{\text {th }}$ International conference on Enterprise Information Systems, Angers, France.

The Enterprise Ontology., http://www.aiai.ed.ac.uk/project/enterprise/enterprise/o ntology.html

Uschold M, King M, Moralee S and Zorgios Y., 1998. "The Enterprise Ontology", The Knowledge Engineering Review, Vol. 13, Special Issue on Putting Ontologies to Use

WorldCom., 2001. "Meeting in America - A study of the Virtual Workforce in 2001", http://emeetings.mci.com/meetingsinamerica/pdf/mia3.pdf

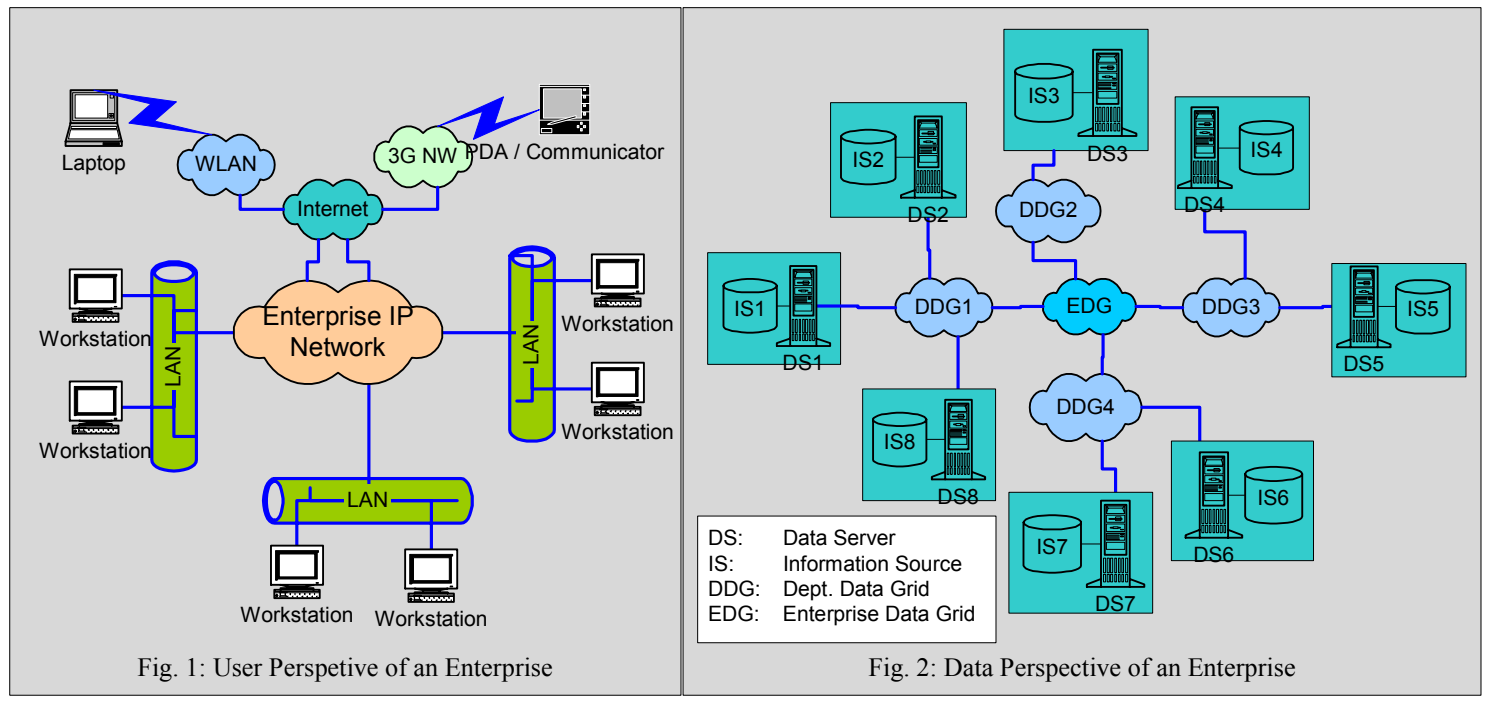



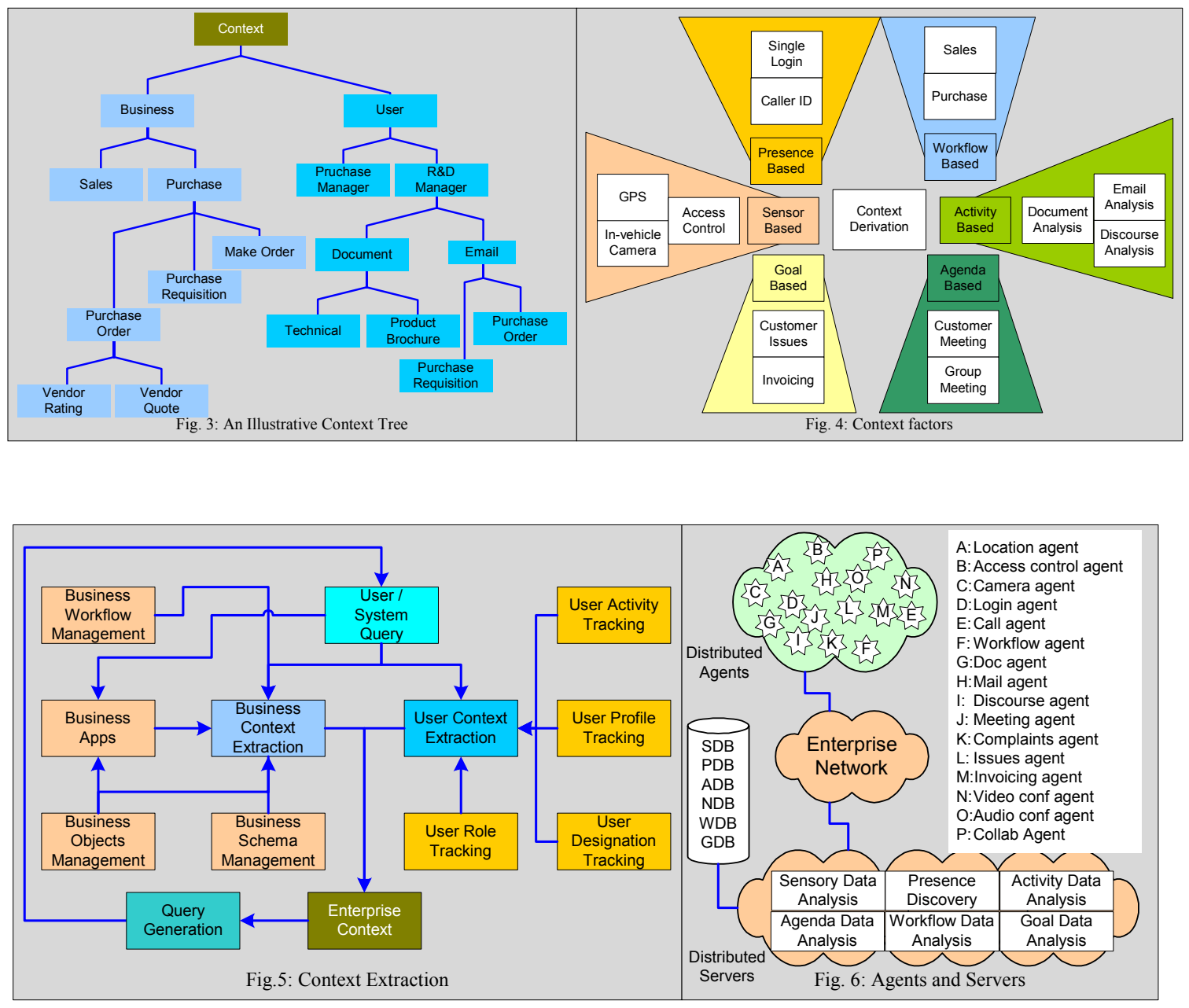

\begin{tabular}{|c|c|c|c|c|}
\hline Agent & $\begin{array}{c}\text { Static / } \\
\text { Dynamic }\end{array}$ & Presistence & Mobility & Location \\
\hline Camera & Static & No & No & Car \\
\hline GPS & Static & No & No & 3G Phone \\
\hline Access control & Static & No & No & Offices \\
\hline Single login & Dynamic & Yes & Mobile & UAD \\
\hline Call & Dynamic & Yes & Mobile & UPD \\
\hline Workflow & Dynamic & Yes & Mobile & UCD \\
\hline Doc analysis & Dynamic & Yes & Mobile & UCD \\
\hline Mail analysis & Dynamic & Yes & Mobile & UAD \\
\hline $\begin{array}{c}\text { Discourse } \\
\text { analysis }\end{array}$ & Dynamic & Yes & Mobile & UcD \\
\hline Agenda & Dynamic & No & Mobile & UCD \\
\hline Goal & Dynamic & Yes & Mobile & UCD \\
\hline
\end{tabular}

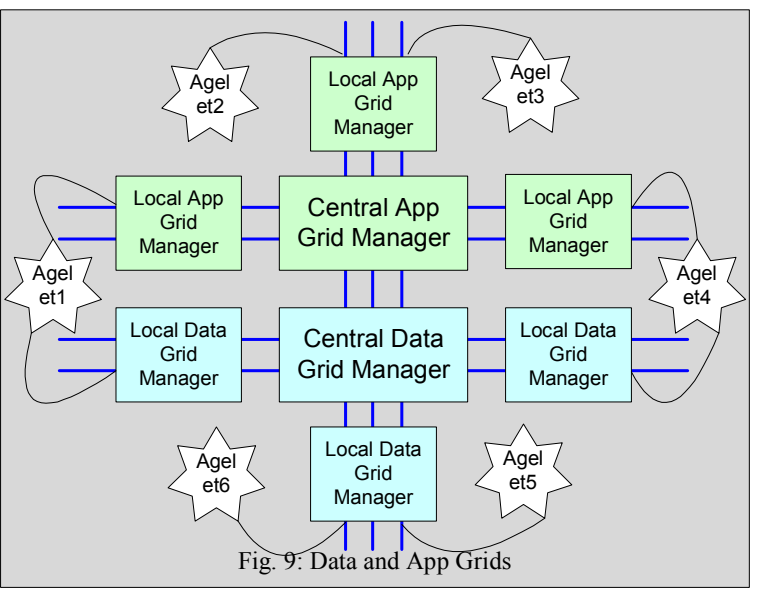




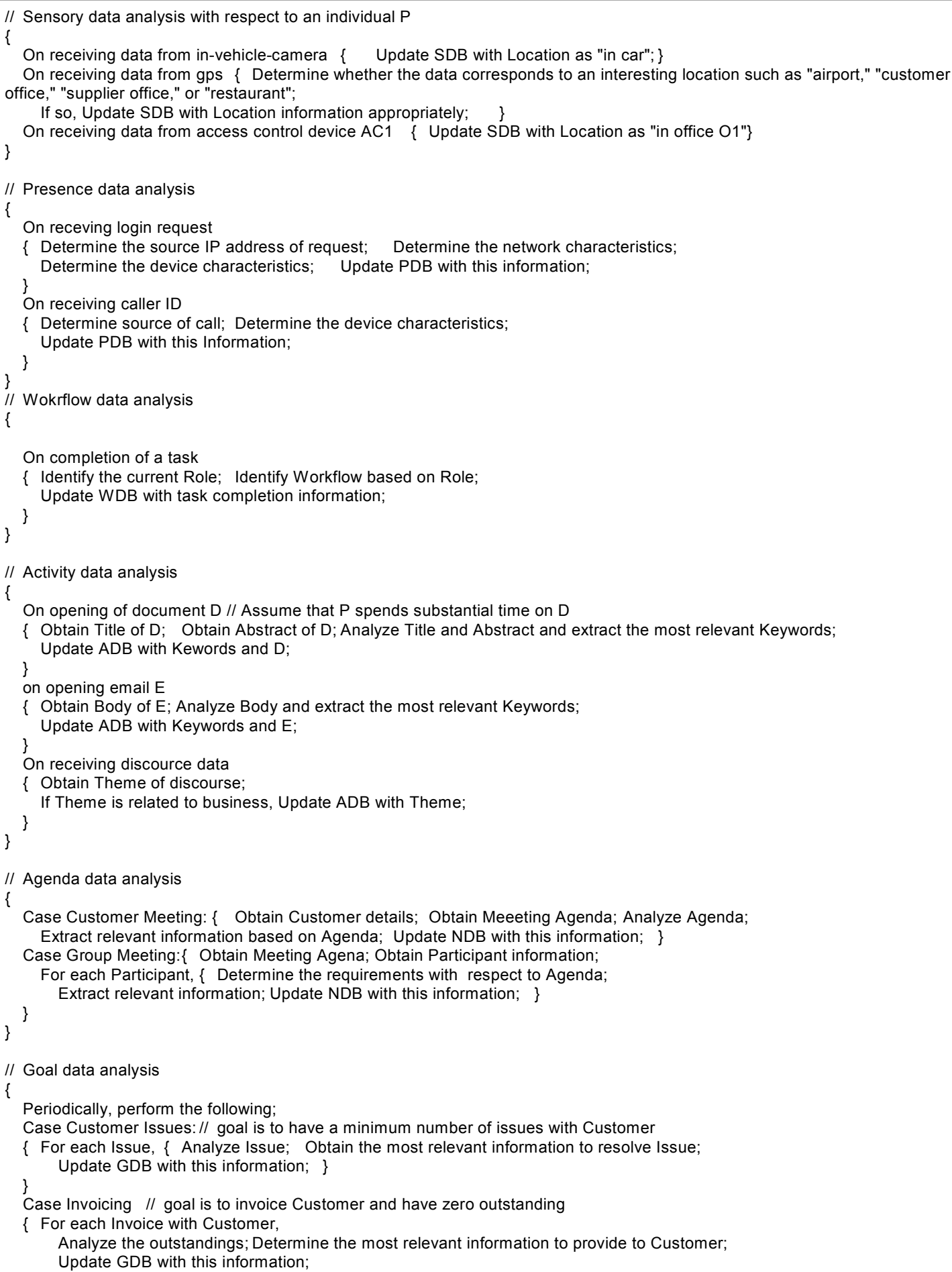

Fig. 8: An Illustrative Set of Context Tracking Procedures 

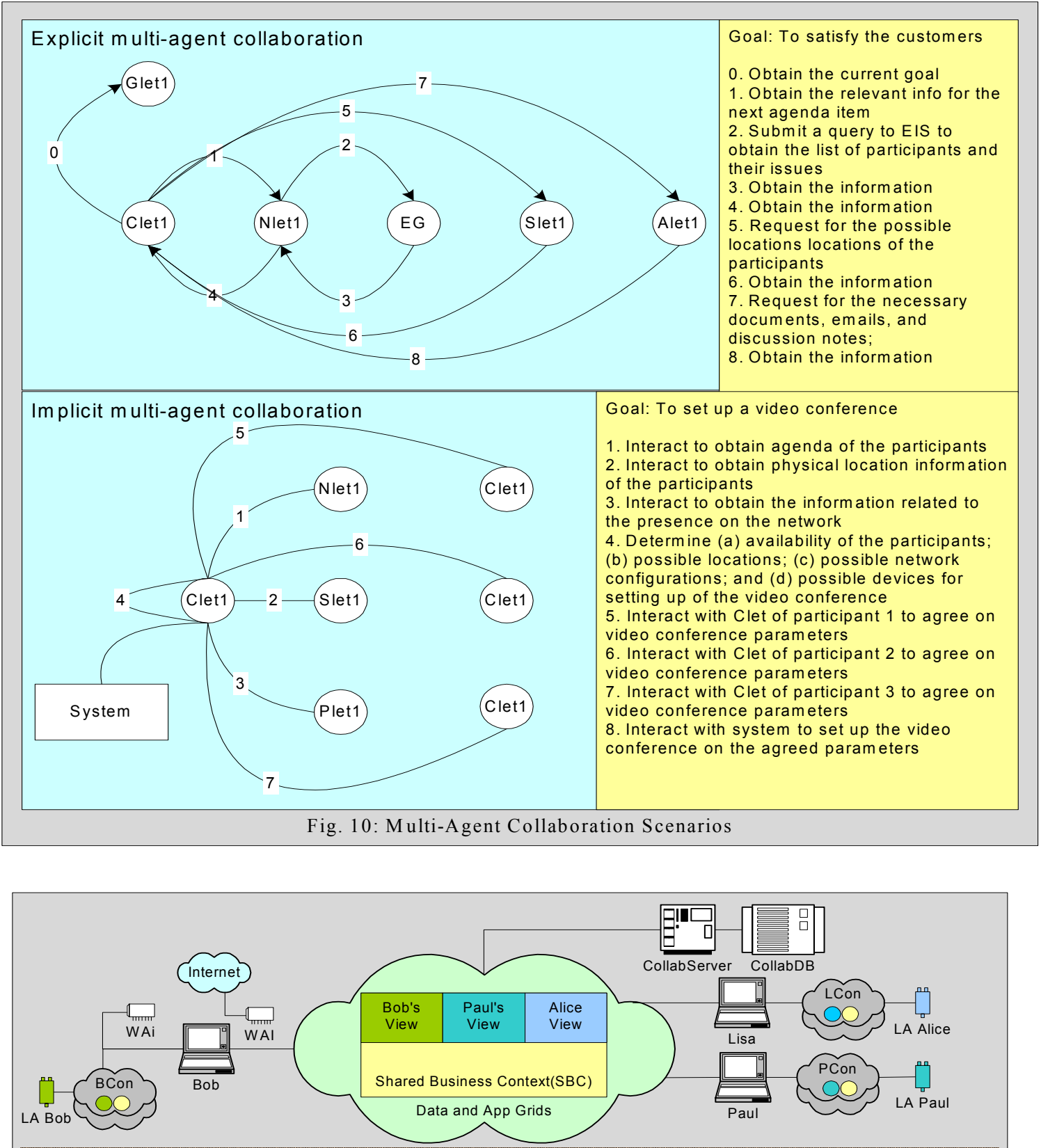

Bob, Paul, and Alice: Collaborators from enterprise E collaborating on business issue participating typically from different geographical locations;

xCon: Context (user context shown as a colored blob and business context shown as a yellow blob) related to business issue associated with collaborator $x$; This context is the result of the study on business issue individually undertaken by the collaborator;

SBC: Shared context space to exchange individual knowledge to other collaborators;

LA y: Local agent of a collaborator; Responsible for maintaining the context of collaborator y;

WAi: Worker agent to perform CAR on internal information sources via data and app grids;

WAI: Worker agent to perform CAR on external information sources via Internet;

Collab Agents associated with each of the participants collaborate to maintain the shared space;

CollabServer: Responsible for initiating and monitoring collaboration sessions; 